\title{
Stability analysis and observer design for a class of nonlinear systems with multiple time-delays
}

\author{
Yali Dong* and Fengwei Yang
}

${ }^{*}$ Correspondence:

dongyl@vip.sina.com

School of Science, Tianjin

Polytechnic University, Tianjin,

300387, China

\begin{abstract}
In this paper, we propose a simple and useful approach to design an observer for multiple time-delays nonlinear systems in a triangular form. By constructing a new Lyapunov-Krasovskii functional and using the differential mean-value theorem, the sufficient conditions for the existence of such an observer are derived, which guarantee that the estimation error converges asymptotically towards zero. The observer gain is independent of the time-delay. A numerical example is provided to illustrate the result.
\end{abstract}

Keywords: time-delay; nonlinear systems; nonlinear observers; asymptotic stability

\section{Introduction}

Time-delay, as well as nonlinearities, is often encountered in various systems which render the control design more difficult [1]. During the past decades, a lot of significant advances have been proposed in stability analysis and feedback control for time-delay systems, e.g., [1-7] and reference therein. Among these schemes, the system states are assumed to be precisely known for the control design, which is not true in some practical cases as some commercial control systems are not equipped with enough sensors. This inspires the issue of observer design for control systems, which is an active research topic in the control community.

Different types of observers have been proposed, e.g., Luenberger observer [8], adaptive observer [9], high-gain observer [10]. The observer design problem for time-delay systems has been widely investigated in the recent years. For time-delay systems, most of the state observation methods developed in the literature concern the linear case; we refer the reader to some recent advances and their extensions [11-13]. However, the problem of state estimation of time-delay systems in the nonlinear case has been rarely studied. For an overview of recent works, see, e.g., [14-16]. In [15], a new approach to the nonlinear observer design problem in the presence of delayed output measurements was presented. The proposed nonlinear observer possesses a state-dependent gain which is computed from the solution of a system of first-order singular partial differential equations. In [18], the authors established a new method for the observer design problem for a class of Lipschitz time-delay systems. The obtained synthesis conditions are expressed in terms of linear matrix inequalities (LMIs) easily tractable and are less restrictive than those obtained in [17]. In [19], the problem of observer design for a class of multi-output nonlinear 
system was considered. A new state observer design methodology for linear time-varying multi-output systems was presented. Furthermore, the same methodology was extended to a class of multi-output nonlinear systems and some sufficient conditions for the existence of the proposed observer were obtained, which guaranteed that the error of state estimation converged asymptotically to zero. For further results on observation of timedelay systems, we refer the reader to [20-23] and the references therein.

In this paper, we investigate observer design for nonlinear systems written in a triangular form. Our main task is to design the observer for a class of nonlinear systems with multiple time-delays. The observer is convergent, whatever the size of the delay. The design method of observer for the class of nonlinear systems with multiple time-delays is proposed, and the gain matrix is obtained. The observer gain is independent of the timedelay. The sufficient conditions are presented, which guarantee that the estimation error converges asymptotically towards zero.

This paper is arranged as follows. In Section 2, the system description and some lemmas are given. In Section 3, we present the observer synthesis method for a class of nonlinear systems with multiple time-delays. In Section 4, we propose an illustrative example in order to show the validity of our method. Finally, some conclusions are given in Section 5 .

The notation used in this paper is fairly standard. Throughout this paper, $R$ stands for the set of real numbers. The notation $A>0(<0)$ means that the matrix $A$ is symmetric and positive definite (negative definite). $A^{T}$ stands for the matrix transpose of matrix $A$. $\|\cdot\|$ denotes the Euclidean norm for a vector or a matrix. $\|\cdot\|_{\infty}$ denotes the infinity norm for a matrix.

\section{System description}

Consider the time-delays nonlinear system given in a lower-triangular form:

$$
\begin{aligned}
& \dot{x}(t)=A x(t)+f(x(t), u(t))+\sum_{j=1}^{k} g_{j}\left(x\left(t-h_{j}\right), u(t)\right), \\
& y=C x(t),
\end{aligned}
$$

where $x(t) \in R^{n}$ is the state vector, $u(t) \in X \subset R^{m}$ is the bounded control input and $y(t) \in R$ is the system output. The delay $h_{j}, j=1,2, \ldots, k$, are constants, and $x(t)=\phi(t)$ for $-h \leq$ $t \leq 0, h=\max _{1 \leq j \leq k}\left\{h_{j}\right\}$. The functions $f(x(t), u(t))$ and $g_{j}(x(t-h), u(t)), j=1,2, \ldots, k$, are nonlinear and are assumed to be smooth, and

$$
\begin{aligned}
A & =\left[\begin{array}{ccccc}
0 & 1 & 0 & \cdots & 0 \\
0 & 0 & 1 & \cdots & 0 \\
\vdots & \vdots & \vdots & \ddots & \vdots \\
0 & 0 & 0 & \cdots & 1 \\
0 & 0 & 0 & \cdots & 0
\end{array}\right] \in R^{n \times n}, \quad C=\left[\begin{array}{lllll}
1 & 0 & 0 & \cdots & 0
\end{array}\right] \in R^{n} \\
f(x(t), u(t)) & =\left[\begin{array}{c}
f_{1}\left(x_{1}(t), u(t)\right) \\
f_{2}\left(x_{1}(t), x_{2}(t), u(t)\right) \\
\vdots \\
f_{n}(x(t), u(t))
\end{array}\right] \in R^{n},
\end{aligned}
$$




$$
g_{j}\left(x\left(t-h_{j}\right), u(t)\right)=\left[\begin{array}{c}
g_{j 1}\left(x_{1}\left(t-h_{j}\right), u(t)\right) \\
g_{j 2}\left(x_{1}\left(t-h_{j}\right), x_{2}\left(t-h_{j}\right), u(t)\right) \\
\vdots \\
g_{j n}\left(x\left(t-h_{j}\right), u(t)\right)
\end{array}\right] \in R^{n}, \quad j=1,2, \ldots, k
$$

To complete the system description, the following assumptions are considered.

Assumption 1 For all $t \geq 0, \alpha \in R^{n}$, the entries of $\frac{\partial f(\alpha, u(t))}{\partial \alpha}$ are bounded.

Assumption 2 For all $t \geq 0, \beta_{j} \in R^{n}$, the entries of $\frac{\partial g_{j}\left(\beta_{j}, u(t)\right)}{\partial \beta_{j}}, j=1,2, \ldots, k$, are bounded.

We set $D(r)=\operatorname{diag}\left(1, r, \ldots, r^{n-1}\right)$ and $\tilde{Q}(r)=r^{-2} D^{-1}(r) \bar{Q} D^{-1}(r)$.

The following lemmas are necessary for the proof of the main statement.

Lemma 1 Let $P(r)$ and $\bar{P}$ be the solutions of the algebraic Riccati equations (AREs):

$$
\begin{aligned}
& P(r) A^{T}+A P(r)-P(r) C^{T} C P(r)+\tilde{Q}(r)=0, \\
& \bar{P} A^{T}+A \bar{P}-\bar{P} C^{T} C \bar{P}+\bar{Q}=0,
\end{aligned}
$$

respectively, where $A$ and $C$ are given in an observable canonical form as in (2), $\bar{Q}$ is any symmetric positive-definite matrix. Then $P(r)$ is positive-definite for $r>0$ and is given by

$$
P(r)=r^{-1} D^{-1}(r) \bar{P} D^{-1}(r) .
$$

Proof Let $\tilde{Q}(r)=B^{T}(r) B(r)$, where $B(r) \in R^{n \times n}$. Since $\tilde{Q}(r)$ is symmetric and positivedefinite for all $r>0$, one gets that $B(r)$ is invertible. It is easy to verify that $\left(A^{T}, C^{T}\right)$ is stabilizable and $\left(B, A^{T}\right)$ is observable. According to ref. [24], we obtain that the matrix $P(r)$ is the unique solution of ARE (3) which is always symmetric and positive-definite for $r>0$.

Using the following properties:

$$
A D(r)=r D(r) A, \quad D(r) A^{T}=r A^{T} D(r), \quad C D(r)=C, \quad D(r) C^{T}=C^{T},
$$

we get

$$
\begin{aligned}
& D^{-1}(r) A=r A D^{-1}(r), \quad A^{T} D^{-1}(r)=r D^{-1}(r) A^{T}, \\
& C=C D^{-1}(r), \quad C^{T}=D^{-1}(r) C^{T} .
\end{aligned}
$$

Pre- and post-multiplying the second ARE in (3) by $r^{-1} D^{-1}(r)$, we have

$$
\begin{aligned}
& r^{-2} D^{-1}(r) \bar{P} A^{T} D^{-1}(r)+r^{-2} D^{-1}(r) A \bar{P} D^{-1}(r) \\
& \quad-r^{-2} D^{-1}(r) \bar{P} C^{T} C \bar{P} D^{-1}(r)+r^{-2} D^{-1}(r) \bar{Q} D^{-1}(r)=0 .
\end{aligned}
$$

Using (4), (5) can be rewritten as

$$
\begin{aligned}
& {\left[r^{-1} D^{-1}(r) \bar{P} D^{-1}(r)\right] A^{T}+A\left[r^{-1} D^{-1}(r) \bar{P} D^{-1}(r)\right]} \\
& \quad-\left[r^{-1} D^{-1}(r) \bar{P} D^{-1}(r)\right] C^{T} C\left[r^{-1} D^{-1}(r) \bar{P} D^{-1}(r)\right]+r^{-2} D^{-1}(r) \bar{Q} D^{-1}(r)=0 .
\end{aligned}
$$


By comparing the last ARE with the first ARE of (3), we conclude that

$$
P(r)=r^{-1} D^{-1}(r) \bar{P} D^{-1}(r) .
$$

Lemma 2 If $L=\left(l_{i j}\right) \in R^{n \times n}$ is a lower-triangular matrix and $D(r)=\operatorname{diag}\left(1, r, \ldots, r^{n-1}\right)$, then the following inequality holds for all $0<r \leq 1$ :

$$
\left\|D(r) L D^{-1}(r)\right\| \leq \delta_{1}+r \delta_{2}
$$

where

$$
\begin{aligned}
& \delta_{1}=\sqrt{n} \max \left\{\left|l_{11}\right|,\left|l_{22}\right|, \ldots,\left|l_{n n}\right|\right\}, \\
& \delta_{2}=\sqrt{n} \max \left\{\left|l_{21}\right|,\left|l_{31}\right|+\left|l_{32}\right|, \ldots,\left|l_{n 1}\right|+\left|l_{n 2}\right|+\cdots+\left|l_{n, n-1}\right|\right\} .
\end{aligned}
$$

Proof Computing the product, we have

$$
\begin{aligned}
D(r) L D^{-1}(r)= & {\left[\begin{array}{lllll}
1 & & & & \\
& r & & & \\
& r^{2} & & \\
& & \ddots & \\
& & & r^{n-1}
\end{array}\right]\left[\begin{array}{ccccc}
l_{11} & 0 & 0 & \cdots & 0 \\
l_{21} & l_{22} & 0 & \cdots & 0 \\
l_{31} & l_{32} & l_{33} & \cdots & 0 \\
\vdots & \vdots & \vdots & \ddots & \vdots \\
l_{n 1} & l_{n 2} & l_{n 3} & \cdots & l_{n n}
\end{array}\right] } \\
& \times\left[\begin{array}{ccccc}
1 & & & \\
& r^{-1} & & & \\
& & r^{-2} & & \\
& & & \ddots & \\
& & & & r^{-n+1}
\end{array}\right] \\
& =\left[\begin{array}{ccccc}
l_{11} & 0 & 0 & \cdots & 0 \\
r l_{21} & l_{22} & 0 & \cdots & 0 \\
r^{2} l_{31} & r l_{32} & l_{33} & \cdots & 0 \\
\vdots & \vdots & \vdots & \ddots & \vdots \\
r^{n-1} l_{n 1} & r^{n-2} l_{n 2} & r^{n-3} l_{n 3} & \cdots & l_{n n}
\end{array}\right] .
\end{aligned}
$$

So, it follows that

$$
\begin{aligned}
\left\|D(r) L D^{-1}(r)\right\| \leq & \sqrt{n}\left\|D(r) L D^{-1}(r)\right\|_{\infty} \\
= & \sqrt{n} \max \left\{\left|l_{11}\right|,\left|l_{22}\right|+\left|r l_{21}\right|,\left|l_{33}\right|+\left|r l_{32}\right|+\left|r^{2} l_{31}\right|, \ldots,\right. \\
& \left.\left|l_{n n}\right|+\left|r^{n-1} l_{n 1}\right|+\left|r^{n-2} l_{n 2}\right|+\cdots+\left|r l_{n, n-1}\right|\right\} \\
= & \sqrt{n} \max \left\{\left|l_{11}\right|,\left|l_{22}\right|+r\left|l_{21}\right|,\left|l_{33}\right|+r\left(\left|l_{32}\right|+\left|r l_{31}\right|\right), \ldots,\right. \\
& \left.\left|l_{n n}\right|+r\left(\left|r^{n-2} l_{n 1}\right|+\left|r^{n-3} l_{n 2}\right|+\cdots+\left|l_{n, n-1}\right|\right)\right\} .
\end{aligned}
$$


When $0<r \leq 1$, we have

$$
\begin{aligned}
\left\|D(r) L D^{-1}(r)\right\| \leq & \sqrt{n} \max \left\{\left|l_{11}\right|,\left|l_{22}\right|+r\left|l_{21}\right|,\left|l_{33}\right|+r\left(\left|l_{32}\right|+\left|l_{31}\right|\right), \ldots\right. \\
& \left.\left|l_{n n}\right|+r\left(\left|l_{n 1}\right|+\left|l_{n 2}\right|+\cdots+\left|l_{n, n-1}\right|\right)\right\} \\
\leq & \delta_{1}+r \delta_{2} .
\end{aligned}
$$

Remark 1 If Assumptions 1 and 2 hold and $0<r \leq 1$, then there are $c_{i}>0, i=1,2, c_{j 3}>0$, $c_{j 4}>0, j=1,2, \ldots, k$, such that

$$
\begin{aligned}
& \left\|D(r) \frac{\partial f(\alpha, u(t))}{\partial \alpha} D^{-1}(r)\right\| \leq c_{1}+r c_{2}, \\
& \left\|D(r) \frac{\partial g_{j}\left(\beta_{j}, u(t)\right)}{\partial \beta_{j}} D^{-1}(r)\right\| \leq c_{j 3}+r c_{j 4}, \quad j=1,2, \ldots, k .
\end{aligned}
$$

Lemma 3 [25] For any real vectors $a, b$ and any matrix $Q>0$ with appropriate dimensions, it follows that

$$
2 a^{T} b \leq a^{T} Q a+b^{T} Q^{-1} b .
$$

Consider the following functional differential equation of retarded type:

$$
\dot{x}(t)=f\left(t, x_{t}\right)
$$

where $x(t) \in R^{n}, f: R \times \mathbb{C} \rightarrow R^{n}$.

Lemma 4 (Lyapunov-Krasovskii stability theorem [1]) Suppose that $f: R \times \mathbb{C} \rightarrow R^{n}$ given in (9) maps every $R \times\left(\right.$ bounded set in $\mathbb{C}$ ) into a bounded set in $R^{n}$, and that $u, v, w: \bar{R}_{+} \rightarrow \bar{R}_{+}$ are continuous nondecreasing functions, where additionally $u(s)$ and $v(s)$ are positive for $s>$ 0 and $u(0)=v(0)=0$. If there exists a continuous differentiable functional $V: R \times \mathbb{C} \rightarrow R$ such that

$$
u(\|\phi(0)\|) \leq V(t, \phi) \leq v\left(\|\phi\|_{c}\right)
$$

and

$$
\dot{V}(t, \phi) \leq-w(\|\phi(0)\|)
$$

then the trivial solution of (9) is uniformly stable. If $w(s)>0$ for $s>0$, then it is uniformly asymptotically stable. In addition, if $\lim _{s \rightarrow \infty} u(s)=\infty$, then it is globally uniformly asymptotically stable.

\section{Observer design}

Now, for the time-delay system described by (1), we propose the following state observer:

$$
\dot{\hat{x}}(t)=A \hat{x}(t)+f(\hat{x}(t), u(t))+\sum_{j=1}^{k} g_{j}\left(\hat{x}\left(t-h_{j}\right), u(t)\right)-L(C \hat{x}(t)-C x(t))
$$


Our aim is to find the gain $L$ such that the estimation error $e(t)=\hat{x}(t)-x(t)$ asymptotically converges towards zero. The estimation error dynamics is governed by

$$
\dot{e}(t)=A e(t)-L C e(t)+\Delta f_{1}+\sum_{j=1}^{k} \Delta g_{j}
$$

where

$$
\begin{aligned}
& \Delta f=f(\hat{x}(t), u(t))-f(x(t), u(t)), \\
& \Delta g_{j}=g_{j}(\hat{x}(t-h), u(t))-g_{j}(x(t-h), u(t)), \quad j=1,2, \ldots, k .
\end{aligned}
$$

In the sequel, we introduce our main contribution which consists of a new feasibility condition for the observer synthesis problem of a class of nonlinear time-delays systems. The convergence analysis is performed by the use of a Lyapunov-Krasovskii functional.

For any symmetric positive-definite matrix $\bar{Q}>0$, let $\bar{P}>0$ be the solutions of the algebraic Riccati equations (AREs):

$$
\bar{P} A^{T}+A \bar{P}-\bar{P} C^{T} C \bar{P}+\bar{Q}=0 .
$$

Theorem 1 Assume that Assumptions 1 and 2 hold and $L=P(r) C^{T}$, where $P(r)=$ $r^{-1} D^{-1}(r) \bar{P} D^{-1}(r)$. Then for any

$$
0<r<\min \left\{1, \frac{\lambda_{\min }\left(\bar{P}^{-1} \bar{Q} \bar{P}^{-1}\right)}{2 \lambda_{\max }\left(\bar{P}^{-1}\right)\left[k+1+\left(c_{1}+c_{2}\right)^{2}\right]}, \min _{1 \leq j \leq k} \frac{\lambda_{\min }\left(\bar{P}^{-1} \bar{Q} \bar{P}^{-1}\right)}{2 k \lambda_{\max }\left(\bar{P}^{-1}\right)\left(c_{j 3}+c_{j 4}\right)^{2}}\right\},
$$

the observer error $e(t)=\hat{x}(t)-x(t)$ that results from (1) and (10) converges asymptotically towards zero.

Proof From Lemma 1, we known that the ARE

$$
P(r) A^{T}+A P(r)-P(r) C^{T} C P(r)+k Q(r)=0,
$$

has the solution

$$
P(r)=r^{-1} D^{-1}(r) \bar{P} D^{-1}(r),
$$

where $Q(r)=(1 / k) r^{-2} D^{-1}(r) \bar{Q} D^{-1}(r)$.

So, we have

$$
A^{T} P^{-1}(r)+P^{-1}(r) A-C^{T} C+k P^{-1}(r) Q(r) P^{-1}(r)=0 .
$$

For positive definite matrices $P^{-1}(r)$, let us consider the Lyapunov-Krasovskii functional candidate:

$$
V(t, e(t))=e^{T}(t) P^{-1}(r) e(t)+\frac{1}{2} \sum_{j=1}^{k} \int_{t-h_{j}}^{t} e^{T}(s) P^{-1}(r) Q(r) P^{-1}(r) e(s) d s .
$$


Then we have

$$
\begin{aligned}
\dot{V}(t, e(t))= & \dot{e}^{T}(t) P^{-1}(r) e(t)+e^{T}(t) P^{-1}(r) \dot{e}(t) \\
& +\frac{1}{2} \sum_{j=1}^{k}\left[e^{T}(t) P^{-1}(r) Q(r) P^{-1}(r) e(t)-e^{T}\left(t-h_{j}\right) P^{-1}(r) Q(r) P^{-1}(r) e\left(t-h_{j}\right)\right] \\
= & e^{T}(t)\left(A^{T} P^{-1}(r)+P^{-1}(r) A-2 C^{T} C+\frac{k}{2} P^{-1}(r) Q(r) P^{-1}(r)\right) e(t) \\
& +2 e^{T}(t) P^{-1}(r)\left(\Delta f+\sum_{j=1}^{k} \Delta g_{j}\right)-\frac{1}{2} \sum_{j=1}^{k} e^{T}\left(t-h_{j}\right) P^{-1}(r) Q(r) P^{-1}(r) e\left(t-h_{j}\right) \\
= & e^{T}(t)\left(-C^{T} C-\frac{k}{2} P^{-1}(r) Q(r) P^{-1}(r)\right) e(t)+2 e^{T}(t) P^{-1}(r)\left(\Delta f+\sum_{j=1}^{k} \Delta g_{j}\right) \\
& -\frac{1}{2} \sum_{j=1}^{k} e^{T}\left(t-h_{j}\right) P^{-1}(r) Q(r) P^{-1}(r) e\left(t-h_{j}\right) .
\end{aligned}
$$

Using the differential mean-value theorem, we can write that

$$
\begin{aligned}
\Delta f & =f(\hat{x}(t), u(t))-f(x(t), u(t)) \\
& =\left.\int_{0}^{1} \frac{\partial f(\alpha, u(t))}{\partial \alpha}\right|_{\alpha=\alpha(\lambda)}(\hat{x}(t)-x(t)) d \lambda, \\
\Delta g_{j} & =g_{j}\left(\hat{x}\left(t-h_{j}\right), u(t)\right)-g_{j}\left(x\left(t-h_{j}\right), u(t)\right) \\
& =\left.\int_{0}^{1} \frac{\partial g_{j}\left(\beta_{j}, u(t)\right)}{\partial \beta_{j}}\right|_{\beta_{j}=\beta_{j}(\lambda)}\left(\hat{x}\left(t-h_{j}\right)-x\left(t-h_{j}\right)\right) d \lambda, \quad j=1,2, \ldots, k,
\end{aligned}
$$

where

$$
\begin{aligned}
& \alpha(\lambda)=x(t)+\lambda(\hat{x}(t)-x(t)), \\
& \beta_{j}(\lambda)=x\left(t-h_{j}\right)+\lambda\left(\hat{x}\left(t-h_{j}\right)-x\left(t-h_{j}\right)\right) .
\end{aligned}
$$

Let us denote

$$
\Psi_{\alpha}(\lambda)=\left.\frac{\partial f(\alpha, u(t))}{\partial \alpha}\right|_{\alpha=\alpha(\lambda)}, \quad \Psi_{\beta_{j}}(\lambda)=\left.\frac{\partial g_{j}\left(\beta_{j}, u(t)\right)}{\partial \beta_{j}}\right|_{\beta_{j}=\beta_{j}(\lambda)} .
$$

This immediately gives

$$
\begin{aligned}
\dot{V}(t, e(t)) \leq & \int_{0}^{1} e^{T}(t)\left(-\frac{k}{2} P^{-1}(r) Q(r) P^{-1}(r)\right) e(t) d \lambda+2 \int_{0}^{1} e^{T}(t) P^{-1}(r) \Psi_{\alpha}(\lambda) e(t) d \lambda \\
& +2 \sum_{j=1}^{k} \int_{0}^{1} e^{T}(t) P^{-1}(r) \Psi_{\beta_{j}}(\lambda) e\left(t-h_{j}\right) d \lambda \\
& -\frac{1}{2} \sum_{j=1}^{k} e^{T}\left(t-h_{j}\right) P^{-1}(r) Q(r) P^{-1}(r) e\left(t-h_{j}\right) .
\end{aligned}
$$


Using Lemma 3, we have

$$
\begin{aligned}
& 2 \int_{0}^{1} e^{T}(t) P^{-1}(r) \Psi_{\alpha}(\lambda) e(t) d \lambda \\
& \quad \leq \int_{0}^{1} e^{T}(t) P^{-1}(r) e(t) d \lambda+\int_{0}^{1} e^{T}(t) \Psi_{\alpha}^{T}(\lambda) P^{-1}(r) \Psi_{\alpha}(\lambda) e(t) d \lambda, \\
& 2 \int_{0}^{1} e^{T}(t) P^{-1}(r) \Psi_{\beta_{j}}(\lambda) e\left(t-h_{j}\right) d \lambda \\
& \quad \leq \int_{0}^{1} e^{T}(t) P^{-1}(r) e(t) d \lambda+\int_{0}^{1} e^{T}\left(t-h_{j}\right) \Psi_{\beta_{j}}^{T}(\lambda) P^{-1}(r) \Psi_{\beta_{j}}(\lambda) e\left(t-h_{j}\right) d \lambda .
\end{aligned}
$$

This implies that

$$
\begin{aligned}
\dot{V}(t, e(t)) \leq & \int_{0}^{1} e^{T}(t)\left(-\frac{k}{2} P^{-1}(r) Q(r) P^{-1}(r)\right) e(t) d \lambda+(k+1) \int_{0}^{1} e^{T}(t) P^{-1}(r) e(t) d \lambda \\
& +\int_{0}^{1} e^{T}(t) \Psi_{\alpha}^{T}(\lambda) P^{-1}(r) \Psi_{\alpha}(\lambda) e(t) d \lambda \\
& +\sum_{j=1}^{k} \int_{0}^{1} e^{T}\left(t-h_{j}\right) \Psi_{\beta_{j}}^{T}(\lambda) P^{-1}(r) \Psi_{\beta_{j}}(\lambda) e\left(t-h_{j}\right) d \lambda \\
& -\frac{1}{2} \sum_{j=1}^{k} e^{T}\left(t-h_{j}\right) P^{-1}(r) Q(r) P^{-1}(r) e\left(t-h_{j}\right) \\
\leq & -\frac{1}{2} \int_{0}^{1} e^{T}(t) D(r) \bar{P}^{-1} \bar{Q} \bar{P}^{-1} D(r) e(t) d \lambda+(k+1) \int_{0}^{1} e^{T}(t) r D(r) \bar{P}^{-1} D(r) e(t) d \lambda \\
& +\int_{0}^{1} e^{T}(t) \Psi_{\alpha}^{T}(\lambda) r D(r) \bar{P}^{-1} D(r) \Psi_{\alpha}(\lambda) e(t) d \lambda \\
& +\sum_{j=1}^{k}\left[\int_{0}^{1} e^{T}\left(t-h_{j}\right) \Psi_{\beta_{j}}^{T}(\lambda) r D(r) \bar{P}^{-1} D(r) \Psi_{\beta_{j}}(\lambda) e\left(t-h_{j}\right) d \lambda\right] \\
& -\frac{1}{2 k} \sum_{j=1}^{k}\left[\int_{0}^{1} e^{T}\left(t-h_{j}\right) D(r) \bar{P}^{-1} \bar{Q}^{-1} D(r) e\left(t-h_{j}\right) d \lambda\right] . \\
&
\end{aligned}
$$

Let $\eta(t)=D(r) e(t)$, we have

$$
\begin{aligned}
\dot{V}(t, e(t)) \leq & -\frac{1}{2} \int_{0}^{1} \eta^{T}(t) \bar{P}^{-1} \bar{Q} \bar{P}^{-1} \eta(t) d \lambda+(k+1) r \int_{0}^{1} \eta^{T}(t) \bar{P}^{-1} \eta(t) d \lambda \\
& +\int_{0}^{1} \eta^{T}(t) D^{-1}(r) \Psi_{\alpha}^{T}(\lambda) r D(r) \bar{P}^{-1} D(r) \Psi_{\alpha}(\lambda) D^{-1}(r) \eta(t) d \lambda \\
& +\sum_{j=1}^{k} \int_{0}^{1} \eta^{T}\left(t-h_{j}\right) D^{-1}(r) \Psi_{\beta_{j}}^{T}(\lambda) r D(r) \bar{P}^{-1} D(r) \Psi_{\beta_{j}}(\lambda) D^{-1}(r) \eta\left(t-h_{j}\right) d \lambda \\
& -\frac{1}{2 k} \sum_{j=1}^{k} \int_{0}^{1} \eta^{T}\left(t-h_{j}\right) \bar{P}^{-1} \bar{Q} \bar{P}^{-1} \eta^{T}\left(t-h_{j}\right) d \lambda \\
\leq & \int_{0}^{1}\left[-\frac{1}{2} \lambda_{\min }\left(\bar{P}^{-1} \bar{Q} \bar{P}^{-1}\right)\right.
\end{aligned}
$$




$$
\begin{aligned}
& \left.+r \lambda_{\max }\left(\bar{P}^{-1}\right)\left(k+1+\left\|D(r) \Psi_{\alpha}(\lambda) D^{-1}(r)\right\|^{2}\right)\right]\|\eta(t)\|^{2} d \lambda \\
& +\sum_{j=1}^{k} \int_{0}^{1}\left[-\frac{1}{2 k} \lambda_{\min }\left(\bar{P}^{-1} \bar{Q} \bar{P}^{-1}\right)\right. \\
& \left.+r \lambda_{\max }\left(\bar{P}^{-1}\right)\left\|D(r) \Psi_{\beta_{j}}(\lambda) D^{-1}(r)\right\|^{2}\right]\left\|\eta\left(t-h_{j}\right)\right\|^{2} d \lambda \\
& \leq \int_{0}^{1}\left[-\frac{1}{2} \lambda_{\min }\left(\bar{P}^{-1} \bar{Q} \bar{P}^{-1}\right)+r \lambda_{\max }\left(\bar{P}^{-1}\right)\left(k+1+\left(c_{1}+r c_{2}\right)^{2}\right)\right]\|\eta(t)\|^{2} d \lambda \\
& +\sum_{j=1}^{k} \int_{0}^{1}\left[-\frac{1}{2 k} \lambda_{\min }\left(\bar{P}^{-1} \bar{Q} \bar{P}^{-1}\right)+r \lambda_{\max }\left(\bar{P}^{-1}\right)\left(c_{j 3}+r c_{j 4}\right)^{2}\right]\left\|\eta\left(t-h_{j}\right)\right\|^{2} d \lambda \\
& \leq \int_{0}^{1}\left[-\frac{1}{2} \lambda_{\min }\left(\bar{P}^{-1} \bar{Q} \bar{P}^{-1}\right)+r \lambda_{\max }\left(\bar{P}^{-1}\right)\left(k+1+\left(c_{1}+c_{2}\right)^{2}\right)\right]\|\eta(t)\|^{2} d \lambda \\
& \quad+\sum_{j=1}^{k} \int_{0}^{1}\left[-\frac{1}{2 k} \lambda_{\min }\left(\bar{P}^{-1} \bar{Q} \bar{P}^{-1}\right)+r \lambda_{\max }\left(\bar{P}^{-1}\right)\left(c_{j 3}+c_{j 4}\right)^{2}\right]\left\|\eta\left(t-h_{j}\right)\right\|^{2} d \lambda .
\end{aligned}
$$

From (13), we have $\dot{V}<0$. According to Lemma 4, we deduce that the observer error converges asymptotically towards zero. This ends the proof of Theorem 1.

Consider the following nonlinear systems:

$$
\begin{aligned}
& \dot{x}(t)=A x(t)+\sum_{j=1}^{k} M_{j} g_{j}\left(x\left(t-h_{j}\right), u(t)\right), \\
& y=C x(t),
\end{aligned}
$$

where $A, C$ and $g_{j}\left(x\left(t-h_{j}\right), u(t)\right)$ are given by (2), and $M_{j}$ is a lower-triangular matrix.

Remark 2 If Assumption 2 holds and $0<r \leq 1$, then there are $\mu_{j 1}>0, \mu_{j 2}>0, j=1,2, \ldots, k$, such that

$$
\left\|D(r) M_{j} \frac{\partial g_{j}\left(\beta_{j}, u(t)\right)}{\partial \beta_{j}} D^{-1}(r)\right\| \leq \mu_{j 1}+r \mu_{j 2}, \quad j=1,2, \ldots, k
$$

Consider the following observer:

$$
\dot{\hat{x}}(t)=A \hat{x}(t)+\sum_{j=1}^{k} M_{j} g_{j}\left(\hat{x}\left(t-h_{j}\right), u(t)\right)-L(C \hat{x}(t)-C x(t)) .
$$

Our aim is to find the gain $L$ such that the estimation error $e(t)=\hat{x}(t)-x(t)$ asymptotically converges towards zero. The estimation error dynamics is governed by

$$
\dot{e}(t)=A e(t)-L C e(t)+\sum_{j=1}^{k} M_{j} \Delta g_{j}
$$


where

$$
\Delta g_{j}=g_{j}(\hat{x}(t-h), u(t))-g_{j}(x(t-h), u(t)), \quad j=1,2, \ldots, k .
$$

Theorem 2 Assume that Assumption 2 holds and $L=P(r) C^{T}$, where $P(r)=r^{-1} D^{-1}(r) \bar{P} \times$ $D^{-1}(r)$. Then for any

$$
0<r<\min \left\{1, \frac{\lambda_{\min }\left(\bar{P}^{-1} \bar{Q} \bar{P}^{-1}\right)}{2 k \lambda_{\max }\left(\bar{P}^{-1}\right)}, \min _{1 \leq j \leq k} \frac{\lambda_{\min }\left(\bar{P}^{-1} \bar{Q} \bar{P}^{-1}\right)}{2 k \lambda_{\max }\left(\bar{P}^{-1}\right)\left(\mu_{j 1}+\mu_{j 2}\right)^{2}}\right\},
$$

the observer error $e(t)=\hat{x}(t)-x(t)$ that results from (16) and (17) converges asymptotically towards zero.

Proof From Lemma 1, we known that the ARE

$$
P(r) A^{T}+A P(r)-P(r) C^{T} C P(r)+k Q(r)=0
$$

has the solution

$$
P(r)=r^{-1} D^{-1}(r) \bar{P} D^{-1}(r),
$$

where $Q(r)=(1 / k) r^{-2} D^{-1}(r) \bar{Q} D^{-1}(r)$.

So, we have

$$
A^{T} P^{-1}(r)+P^{-1}(r) A-C^{T} C+k P^{-1}(r) Q(r) P^{-1}(r)=0 .
$$

For positive definite matrices $P^{-1}(r)$, let us consider the Lyapunov-Krasovskii functional candidate

$$
V(t, e(t))=e^{T}(t) P^{-1}(r) e(t)+\frac{1}{2} \sum_{j=1}^{k} \int_{t-h_{j}}^{t} e^{T}(s) P^{-1}(r) Q(r) P^{-1}(r) e(s) d s .
$$

Then we have

$$
\begin{aligned}
\dot{V}(t, e(t))= & \dot{e}^{T}(t) P^{-1}(r) e(t)+e^{T}(t) P^{-1}(r) \dot{e}(t)+\frac{1}{2} \sum_{j=1}^{k}\left[e^{T}(t) P^{-1}(r) Q(r) P^{-1}(r) e(t)\right. \\
& \left.-e^{T}\left(t-h_{j}\right) P^{-1}(r) Q(r) P^{-1}(r) e\left(t-h_{j}\right)\right] \\
= & e^{T}(t)\left(A^{T} P^{-1}(r)+P^{-1}(r) A-2 C^{T} C+\frac{k}{2} P^{-1}(r) Q(r) P^{-1}(r)\right) e(t) \\
& +2 e^{T}(t) P^{-1}(r)\left(\sum_{j=1}^{k} M_{j} \Delta g_{j}\right)-\frac{1}{2} \sum_{j=1}^{k} e^{T}\left(t-h_{j}\right) P^{-1}(r) Q(r) P^{-1}(r) e\left(t-h_{j}\right) \\
= & e^{T}(t)\left(-C^{T} C-\frac{k}{2} P^{-1}(r) Q(r) P^{-1}(r)\right) e(t)+2 e^{T}(t) P^{-1}(r)\left(\sum_{j=1}^{k} M_{j} \Delta g_{j}\right) \\
& -\frac{1}{2} \sum_{j=1}^{k} e^{T}\left(t-h_{j}\right) P^{-1}(r) Q(r) P^{-1}(r) e\left(t-h_{j}\right) .
\end{aligned}
$$


Using the differential mean-value theorem, we can write that

$$
\begin{aligned}
\Delta g_{j} & =g_{j}\left(\hat{x}\left(t-h_{j}\right), u(t)\right)-g_{j}\left(x\left(t-h_{j}\right), u(t)\right) \\
& =\left.\int_{0}^{1} \frac{\partial g_{j}\left(\beta_{j}, u(t)\right)}{\partial \beta_{j}}\right|_{\beta_{j}=\beta_{j}(\lambda)}\left(\hat{x}\left(t-h_{j}\right)-x\left(t-h_{j}\right)\right) d \lambda, \quad j=1,2, \ldots, k,
\end{aligned}
$$

where

$$
\beta_{j}(\lambda)=x\left(t-h_{j}\right)+\lambda\left(\hat{x}\left(t-h_{j}\right)-x\left(t-h_{j}\right)\right) .
$$

Let us denote

$$
\Psi_{\beta_{j}}(\lambda)=\left.\frac{\partial g_{j}\left(\beta_{j}, u(t)\right)}{\partial \beta_{j}}\right|_{\beta_{j}=\beta_{j}(\lambda)} .
$$

This immediately gives

$$
\begin{aligned}
\dot{V}(t, e(t)) \leq & \int_{0}^{1} e^{T}(t)\left(-\frac{k}{2} P^{-1}(r) Q(r) P^{-1}(r)\right) e(t) d \lambda \\
& +2 \sum_{j=1}^{k} \int_{0}^{1} e^{T}(t) P^{-1}(r) M_{j} \Psi_{\beta_{j}}(\lambda) e\left(t-h_{j}\right) d \lambda \\
& -\frac{1}{2} \sum_{j=1}^{k} e^{T}\left(t-h_{j}\right) P^{-1}(r) Q(r) P^{-1}(r) e\left(t-h_{j}\right) .
\end{aligned}
$$

Using Lemma 3, we have

$$
\begin{aligned}
& 2 \int_{0}^{1} e^{T}(t) P^{-1}(r) M_{j} \Psi_{\beta_{j}}(\lambda) e\left(t-h_{j}\right) d \lambda \\
& \leq \int_{0}^{1} e^{T}(t) P^{-1}(r) e(t) d \lambda \\
& \quad+\int_{0}^{1} e^{T}\left(t-h_{j}\right) \Psi_{\beta_{j}}^{T}(\lambda) M_{j}^{T} P^{-1}(r) M_{j} \Psi_{\beta_{j}}(\lambda) e\left(t-h_{j}\right) d \lambda .
\end{aligned}
$$

This implies that

$$
\begin{aligned}
\dot{V}(t, e(t)) \leq & \int_{0}^{1} e^{T}(t)\left(-\frac{k}{2} P^{-1}(r) Q(r) P^{-1}(r)\right) e(t) d \lambda+k \int_{0}^{1} e^{T}(t) P^{-1}(r) e(t) d \lambda \\
& +\sum_{j=1}^{k} \int_{0}^{1} e^{T}\left(t-h_{j}\right) \Psi_{\beta_{j}}^{T}(\lambda) M_{j}^{T} P^{-1}(r) M_{j} \Psi_{\beta_{j}}(\lambda) e\left(t-h_{j}\right) d \lambda \\
& -\frac{1}{2} \sum_{j=1}^{k} e^{T}\left(t-h_{j}\right) P^{-1}(r) Q(r) P^{-1}(r) e\left(t-h_{j}\right) \\
\leq & -\frac{1}{2} \int_{0}^{1} e^{T}(t) D(r) \bar{P}^{-1} \bar{Q} \bar{P}^{-1} D(r) e(t) d \lambda+k \int_{0}^{1} e^{T}(t) r D(r) \bar{P}^{-1} D(r) e(t) d \lambda
\end{aligned}
$$




$$
\begin{aligned}
& +\sum_{j=1}^{k}\left[\int_{0}^{1} e^{T}\left(t-h_{j}\right) \Psi_{\beta_{j}}^{T}(\lambda) M_{j}^{T} r D(r) \bar{P}^{-1} D(r) M_{j} \Psi_{\beta_{j}}(\lambda) e\left(t-h_{j}\right) d \lambda\right. \\
& \left.-\int_{0}^{1} \frac{1}{2 k} e^{T}\left(t-h_{j}\right) D(r) \bar{P}^{-1} \bar{Q} \bar{P}^{-1} D(r) e\left(t-h_{j}\right) d \lambda\right] .
\end{aligned}
$$

Let $\eta(t)=D(r) e(t)$, we have

$$
\begin{aligned}
\dot{V}(t, e(t)) \leq & -\frac{1}{2} \int_{0}^{1} \eta^{T}(t) \bar{P}^{-1} \bar{Q} \bar{P}^{-1} \eta(t) d \lambda+k r \int_{0}^{1} \eta^{T}(t) \bar{P}^{-1} \eta(t) d \lambda \\
& +\sum_{j=1}^{k} \int_{0}^{1} \eta^{T}\left(t-h_{j}\right) D^{-1}(r) \Psi_{\beta_{j}}^{T}(\lambda) M_{j}^{T} r \\
& \times D(r) \bar{P}^{-1} D(r) M_{j} \Psi_{\beta_{j}}(\lambda) D^{-1}(r) \eta\left(t-h_{j}\right) d \lambda \\
& -\frac{1}{2 k} \sum_{j=1}^{k} \int_{0}^{1} \eta^{T}\left(t-h_{j}\right) \bar{P}^{-1} \bar{Q} \bar{P}^{-1} \eta^{T}\left(t-h_{j}\right) d \lambda \\
\leq & \int_{0}^{1}\left[-\frac{1}{2} \lambda_{\min }\left(\bar{P}^{-1} \bar{Q} \bar{P}^{-1}\right)+k r \lambda_{\max }\left(\bar{P}^{-1}\right)\right]\|\eta(t)\|^{2} d \lambda \\
& +\sum_{j=1}^{k} \int_{0}^{1}\left[-\frac{1}{2 k} \lambda_{\min }\left(\bar{P}^{-1} \bar{Q} \bar{P}^{-1}\right)\right. \\
& \left.+r \lambda_{\max }\left(\bar{P}^{-1}\right)\left\|D(r) M_{j} \Psi_{\beta_{j}}(\lambda) D^{-1}(r)\right\|^{2}\right]\left\|\eta\left(t-h_{j}\right)\right\|^{2} d \lambda \\
\leq & \int_{0}^{1}\left[-\frac{1}{2} \lambda_{\min }\left(\bar{P}^{-1} \bar{Q} \bar{P}^{-1}\right)+k r \lambda_{\max }\left(\bar{P}^{-1}\right)\right]\|\eta(t)\|^{2} d \lambda \\
& +\sum_{j=1}^{k} \int_{0}^{1}\left[-\frac{1}{2 k} \lambda_{\min }\left(\bar{P}^{-1} \bar{Q} \bar{P}^{-1}\right)+r \lambda_{\max }\left(\bar{P}^{-1}\right)\left(\mu_{j 1}+r \mu_{j 2}\right)^{2}\right]\left\|\eta\left(t-h_{j}\right)\right\|^{2} d \lambda \\
\leq & \int_{0}^{1}\left[-\frac{1}{2} \lambda_{\min }\left(\bar{P}^{-1} \bar{Q} \bar{P}^{-1}\right)+k r \lambda_{\max }\left(\bar{P}^{-1}\right)\right]\|\eta(t)\|^{2} d \lambda \\
& +\sum_{j=1}^{k} \int_{0}^{1}\left[-\frac{1}{2 k} \lambda_{\min }\left(\bar{P}^{-1} \bar{Q} \bar{P}^{-1}\right)+r \lambda_{\max }\left(\bar{P}^{-1}\right)\left(\mu_{j 1}+\mu_{j 2}\right)^{2}\right]\left\|\eta\left(t-h_{j}\right)\right\|^{2} d \lambda
\end{aligned}
$$

From (19), we have $\dot{V}<0$. This ends the proof of Theorem 2 .

Remark 3 In (16), the nonlinear term is the function of $u(t)$ and $x\left(t-h_{j}\right), j=1,2, \ldots, k$. But it does not contain $x(t)$. If $f(x(t), u(t))=0$, then (1) can be written as

$$
\begin{aligned}
& \dot{x}(t)=A x(t)+\sum_{j=1}^{k} g_{j}\left(x\left(t-h_{j}\right), u(t)\right), \\
& y=C x(t) .
\end{aligned}
$$

When $M_{j}=I, j=1,2, \ldots, k,(16)$ becomes (22). So, (22) is the special case of (16). 
Consider the following time-delay system:

$$
\begin{aligned}
& \dot{x}(t)=A x(t)+A_{1} x(t)+\sum_{j=1}^{k} B_{j} x\left(t-h_{j}\right)+\omega(u(t)), \\
& y=C x(t),
\end{aligned}
$$

where $A$ and $C$ are defined as in (2). $A_{1}=\left(a_{i j}\right)$ and $B_{j}=\left(b_{i k}^{j}\right), j=1,2, \ldots, k$, are real and lower-triangular matrices and $\omega(u(t))$ is an input-injection vector of dimension $n$.

From Lemma 2, we have

$$
\begin{aligned}
& \left\|D(r) A_{1} D^{-1}(r)\right\| \leq v_{1}+r v_{2}, \\
& \left\|D(r) B_{j} D^{-1}(r)\right\| \leq v_{j 3}+r v_{j 4}, \quad j=1,2, \ldots, k,
\end{aligned}
$$

where

$$
\begin{aligned}
& v_{1}=\sqrt{n} \max \left\{\left|a_{11}\right|,\left|a_{22}\right|, \ldots,\left|a_{n n}\right|\right\}, \\
& v_{2}=\sqrt{n} \max \left\{\left|a_{21}\right|,\left|a_{31}\right|+\left|a_{32}\right|, \ldots,\left|a_{n 1}\right|+\left|a_{n 2}\right|+\cdots+\left|a_{n, n-1}\right|\right\}, \\
& v_{j 3}=\sqrt{n} \max \left\{\left|b_{11}^{j}\right|,\left|b_{22}^{j}\right|, \ldots,\left|b_{n n}^{j}\right|\right\}, \\
& v_{j 4}=\sqrt{n} \max \left\{\left|b_{21}^{j}\right|,\left|b_{31}^{j}\right|+\left|b_{32}^{j}\right|, \ldots,\left|b_{n 1}^{j}\right|+\left|b_{n 2}^{j}\right|+\cdots+\left|b_{n, n-1}^{j}\right|\right\} .
\end{aligned}
$$

Consider the following observer:

$$
\dot{\hat{x}}(t)=A \hat{x}(t)+A_{1} \hat{x}(t)+\sum_{j=1}^{k} B_{j} \hat{x}\left(t-h_{j}\right)-L(C \hat{x}(t)-C x(t))+\omega(u(t)) .
$$

The estimation error is $e(t)=\hat{x}(t)-x(t)$. The estimation error dynamics is governed by

$$
\dot{e}(t)=\left(A+A_{1}-L C\right) e(t)+\sum_{j=1}^{k} B_{j} e\left(t-h_{j}\right) .
$$

Corollary 1 Consider the nonlinear system (23). Assume that $L=P(r) C^{T}$, where $P(r)=$ $r^{-1} D^{-1}(r) \bar{P} D^{-1}(r)$. Then for any

$$
0<r<\min \left\{1, \frac{\lambda_{\min }\left(\bar{P}^{-1} \bar{Q} \bar{P}^{-1}\right)}{2 \lambda_{\max }\left(\bar{P}^{-1}\right)\left[k+1+\left(v_{1}+v_{2}\right)^{2}\right]}, \min _{1 \leq j \leq k} \frac{\lambda_{\min }\left(\bar{P}^{-1} \bar{Q} \bar{P}^{-1}\right)}{2 k \lambda_{\max }\left(\bar{P}^{-1}\right)\left[\left(v_{j 3}+v_{j 4}\right)^{2}\right]}\right\},
$$

the estimation error $e(t)=\hat{x}(t)-x(t)$ that results from (23) and (26) converges asymptotically towards zero.

Proof The matrices $A$ and $B_{j}$ can be seen as the matrix Jacobian. Therefore, the proof becomes straightforward as it was developed before.

Remark 4 Those results obtained can be extended to multiple time-delays nonlinear systems in upper-triangular form. 
Remark 5 In [26], the sufficient conditions which guarantee that the estimation error converges asymptotically towards zero are given in terms of a linear matrix inequality. Comparing with [26], our results are less conservative and more convenient to use.

\section{Numerical example}

Let us consider the time delay system

$$
\begin{aligned}
& \dot{x}_{1}(t)=x_{2}(t)+\sin \left(x_{1}(t-1)+u(t)\right), \\
& \dot{x}_{2}(t)=-0.15 \sin \left(x_{2}(t-0.5)\right), \\
& y(t)=x_{1}(t),
\end{aligned}
$$

where

$$
\begin{aligned}
& A=\left[\begin{array}{ll}
0 & 1 \\
0 & 0
\end{array}\right], \quad C=\left[\begin{array}{ll}
1 & 0
\end{array}\right], \quad h_{1}=1, \quad h_{2}=0.5, \\
& x(t)=\left(\begin{array}{l}
x_{1}(t) \\
x_{2}(t)
\end{array}\right), \quad M_{1}=M_{2}=I, \\
& g_{1}\left(x\left(t-h_{1}\right), u(t)\right)=\left(\begin{array}{c}
\sin \left(x_{1}(t-1)+u(t)\right) \\
0
\end{array}\right), \\
& g_{2}\left(x\left(t-h_{2}\right), u(t)\right)=\left(\begin{array}{c}
0 \\
-0.15 \sin \left(x_{2}(t-0.5)\right)
\end{array}\right) .
\end{aligned}
$$

Take

$$
\bar{Q}=\left[\begin{array}{ll}
2 & 0 \\
0 & 1
\end{array}\right] .
$$

Solving the following equation:

$$
\bar{P} A^{T}+A \bar{P}-\bar{P} C^{T} C \bar{P}+\bar{Q}=0,
$$

we get

$$
\bar{P}=\left[\begin{array}{ll}
2 & 1 \\
1 & 2
\end{array}\right], \quad \bar{P}^{-1}=\frac{1}{3}\left[\begin{array}{cc}
2 & -1 \\
-1 & 2
\end{array}\right] .
$$

It is easy to obtain that $\mu_{11}=\mu_{12}=1, \mu_{21}=\mu_{22}=0.5$. Let $r=0.025, D(r)=\left[\begin{array}{ll}1 & 0 \\ 0 & r\end{array}\right]$. It is easy to verify that (19) holds.

We get

$$
L=\left.r^{-1} D^{-1}(r) \bar{P} D^{-1}(r) C^{T}\right|_{r=0.025}=\left[\begin{array}{c}
80 \\
1,600
\end{array}\right] .
$$


The observer is given by

$$
\begin{aligned}
& \dot{\hat{x}}_{1}(t)=\hat{x}_{2}(t)+\sin \left(\hat{x}_{1}(t-1)+u(t)\right)-80\left(\hat{x}_{1}(t)-x_{1}(t)\right), \\
& \dot{\hat{x}}_{2}(t)=-0.15 \sin \left(\hat{x}_{2}(t-0.5)\right)-1,600\left(\hat{x}_{1}(t)-x_{1}(t)\right) .
\end{aligned}
$$

According to Theorem 2, the estimation error $e(t)=\hat{x}(t)-x(t)$ converges asymptotically towards zero.

\section{Conclusion}

The main purpose of this paper is to offer a systematic algorithm for designing an observer for a class of nonlinear systems with multiple time-delays. By using an improved Lyapunov-Krasovskii functional and the differential mean-value theorem, we present the sufficient conditions for the existence of the observer, which guarantee that the estimation error converges asymptotically towards zero. The new design plays an important role in obtaining a nonrestrictive synthesis condition and rendering our approach application to a broader class of systems, namely the class of nonlinear time-delay systems in a lowertriangular form. The proposed design is valid whatever the size of the delay. Finally, the efficiency of the proposed method is shown by a numerical example.

\section{Competing interests}

The authors declare that they have no competing interests.

\section{Authors' contributions}

YD carried out the main part of this manuscript. FY participated in the discussion and gave the example. All authors read and approved the final manuscript.

\section{Acknowledgements}

This work was supported by the Natural Science Foundation of Tianjin under Grant 11JCYBJC06800.

Received: 5 December 2012 Accepted: 7 May 2013 Published: 23 May 2013

\section{References}

1. Gu, K, Chen, J, Kharitonov, VL: Stability of Time-Delay Systems. Birkhäuser, Boston (2003)

2. Dong, $Y$, Wang, $X, M e i, S, L i, W$ : Exponential stabilization of nonlinear uncertain systems with time-varying delay. J. Eng. Math. 77, 225-237 (2012)

3. Ge, SS, Tee, KP: Approximation-based control of nonlinear MIMO time-delay systems. Automatica 34, 31-43 (2007)

4. Dong, Y, Liu, J: Exponential stabilization of uncertain nonlinear time-delay systems. Adv. Differ. Equ. 2012, 1-11 (2012) doi:10.1186/1687-1847-2012-180

5. Diblik, J, Khusainov, DY, Rủžičková, M: Controllability of linear discrete systems with constant coefficients and pure delay. SIAM J. Control Optim. 47(3), 1140-1149 (2008). doi:10.1137/070689085

6. Diblik, J, Khusainov, DY, Lukáčová, J, Rưžičková, M: Control of oscillating systems with a single delay. Adv. Differ. Equ. 2010, Article ID 108218 (2010). doi:10.1155/2010/108218

7. Baštinec, J, Diblík, J, Khusainov, DY, Ryvolová, A: Exponential stability and estimation of solutions of linear differential systems of neutral type with constant coefficients. Bound. Value Probl. 2010, Article ID 956121 (2010). doi:10.1155/2010/956121

8. Shim, H, Son, Yl, Seo, JH: Semi-global observer for multi-output nonlinear systems. Syst. Control Lett. 42, 233-244 (2001)

9. Dong, Y, Mei, S: Adaptive observer for a class of nonlinear systems. Acta Autom. Sin. 33, 1081-1084 (2007)

10. Tornambe, A: High-gain observers for non-linear systems. Int. J. Syst. Sci. 23, 1475-1489 (1992)

11. Boutayeb, M, Darouach, M: Observers for discrete-time systems with multiple delays. IEEE Trans. Autom. Control 46, 746-750 (2001)

12. Boutayeb, M: Observers design for linear time-delay systems. Syst. Control Lett. 44, 103-109 (2001)

13. Darouach, M: Linear functional observers for systems with delays in state variables: the discrete-time case. IEEE Trans. Autom. Control 50, 228-233 (2005)

14. Trinh, H, Aldeen, $M$, Nahavandi, S: An observer design procedure for a class of nonlinear time-delay systems. Comput. Electr. Eng. 30, 61-71 (2004)

15. Kazantzis, N, Wright, RA: Nonlinear observer design in the presence of delayed output measurements. Syst. Control Lett. 54, 877-886 (2005)

16. Dong, Y, Liu, J, Mei, S: Observer design for a class of nonlinear discrete-time systems with time-delay. Kybernetika 49 , 342-359 (2013) 
17. Aggoune, W, Boutayeb, M, Darouach, M: Observers design for a class of nonlinear systems with time-varying delay. In: CDC'1999, Phoenix, Arizona USA, December, 3, pp. 2912-2913 (1999)

18. Zemouche, A, Boutayeb, M, Bara, Gl: On observers design for nonlinear time-delay systems. In: 2006 American Control Conference ACC'06, Minneapolis, Minnesota, USA, June (2006)

19. Dong, Y, Mei, S: State observers for a class of multi-output nonlinear dynamic systems. Nonlinear Anal., Theory Methods Appl. 74, 4738-4745 (2011)

20. Ibrir, S, Xie, WF, Su, C-Y: Observer design for discrete-time systems subject to time-delay nonlinearities. Int. J. Syst. Sci. 37, 629-641 (2006)

21. Boutayeb, M: Observer design for linear time-delay systems. Syst. Control Lett. 44, 103-109 (2001)

22. Wang, Z, Goodall, DP, Burnham, KJ: On designing observers for time-delay systems with nonlinear disturbances. Int. J. Control 75, 803-811 (2002)

23. Germani, A, Manes, C, Pepe, P: A new approach to state observation of nonlinear systems with delayed output. IEEE Trans. Autom. Control 47, 96-101 (2002)

24. Ni, M: Existence condition on solutions to the algebraic Riccati equation. Acta Autom. Sin. 34, 85-87 (2008)

25. $\mathrm{Wu}, \mathrm{H}$, Mizukami, $\mathrm{K}$ : Exponential stability of a class of nonlinear dynamics systems with uncertainties. Syst. Control Lett. 21, 307-313 (1993)

26. Zemouche, A, Boutayeb, M, Bara, Gl: Observers for a class of Lipschitz systems with extension to $H_{\infty}$ performance analysis. Syst. Control Lett. 57, 18-27 (2008)

doi:10.1186/1687-1847-2013-147

Cite this article as: Dong and Yang: Stability analysis and observer design for a class of nonlinear systems with multiple time-delays. Advances in Difference Equations 2013 2013:147.

\section{Submit your manuscript to a SpringerOpen ${ }^{\circ}$ journal and benefit from:}

- Convenient online submission

- Rigorous peer review

- Immediate publication on acceptance

- Open access: articles freely available online

- High visibility within the field

- Retaining the copyright to your article 